\title{
PERFORMANCE OPTIMIZATION OF ENERGY RECOVERY DEVICE BASED ON PAT WITH GUIDE VANE
}

\author{
Shi, H. X.*; Chai, L. P. ; Su, X. Z.** \& Jaini, R. ${ }^{* * *}$ \\ * School of Mechanical Engineering, Hefei University of Technology, Tunxi Road No.193, \\ Hefei 230009, Anhui, China \\ *** Hefei Huasheng Pumps \& Valves Co., Ltd., Jinhuai Road 06, Hefei 230009, Anhui, China \\ ${ }^{* * * *}$ Research and Production Centre "EIP", M.G. Road IFFCO Chowk, Gurugram, Haryana 122002, India \\ E-Mail: suxiaozhen870727@126.com
}

\begin{abstract}
Among the technologies for middle-pressure and low-pressure energy recovery, the influence of guide vane is generally neglected in pump as turbine (PAT) based on centrifugal pumps. For high-pressure energy recovery technology, only few studies have been conducted on PAT with guide vane, and the dynamic and static flow laws between the inverse impeller and guide vanes remain unclear. In this study, by optimizing the key geometric parameters of the impeller with forward-curved blades, the influence of different parameters on its performance was investigated. Results show that when the number of blades is 10 at the same flow rate, the highest efficiency is achieved and the internal flow becomes stable. With the same number of blades, the total head increases gradually with the increase of flow rate, whereas the efficiency increases initially and then decreases. Under the same head, the PAT model with blade outlet angle achieves the highest efficiency, power and the best hydraulic performance. Conclusions obtained in this study have important value for implications to optimize the cavitation of multi-stage PAT.

(Received in March 2018, accepted in June 2018. This paper was with the authors 1 month for 1 revision.)
\end{abstract}

Key Words: Pump as Turbine (PAT), Impeller with Forward-Curved Blades, Numerical Simulation, Parameter Optimization

\section{INTRODUCTION}

Pump as turbine (PAT) is a type of energy recovery device and is extensively used in many fields, such as petrochemical engineering, electric power, and seawater desalination. It is based on the inversion of single-stage or multi-stage centrifugal pump. In other words, highpressure fluid flows into the pump from the pump outlet and flows out from the pump inlet to provide power to the blade; the pressure energy is transformed into the mechanical energy to drive the rotation of the electromotor and redundant energy is regained. Previous studies have focused on PAT units of middle-pressure and low-pressure energy recovery [1]. In structure selection and design of pumps based on the principle of solid mechanics, the hydrogen feeding pump is driven by the PAT and asynchronous motor together. The electric motor is installed between the pump and PAT. Meanwhile, a one-way clutch is assembled between the PAT and the motor. Both the pump and PAT is double case multistage cylindrical centrifugal pump. This design can improve the operational stability effectively. Some studies are focused on the internal flow characteristics and performance optimization of single-stage PAT models based on hydrodynamic principles, such as the analysis of the slip velocity characteristics on pump inversion PAT and the influence of the structural changes of forward- and backwardcurved blades on internal flow characteristics etc. [2].

With the continuous development of PAT technology, multi-stage PAT devices with high recovery power (i.e., $500 \mathrm{~kW}$ ) have attracted increasing attention. In comparison with the single-stage PAT, the addition of a guide vane results in the fluid flowing into the subsequent level conveniently. Existing studies have focused on the conversion coefficient of PAT working conditions and pump working conditions of the single-stage. Theoretical foundation 
is insufficient especially for excellent multi-stage PAT models with high recovery power [3-5], In addition, only few studies have been conducted on influence of guide vane on the internal flow of the impeller, and the unstable flow mechanism induced by their dynamicstatic coupling. Therefore, predicting the internal flow in the PAT with guide vane accurately, determining the coupling mechanism between the forward-curved blades and the guide vane, and optimizing key influencing factors (e.g., the number of blades, blade outlet angle and wrapping angle of the blade on its performance are effective approaches to improve the performance of the PAT with guide vane and address the aforementioned problems rapidly.

\section{STATE OF THE ART}

Scholars all over the world have conducted considerable studies on energy source. Several industrial residual pressure recovery plants have elicited growing attention. PAT-based technology of residual pressure recovery is becoming an academic research hotspot. Researchers have conducted several studies on this topic. Derakhshan et al. [6, 7] investigated a PAT model in various rotational speeds by theoretical analysis and model test and optimized geometric parameters of impeller by combining gradient algorithms. However, the influence of forward-curved blades on the performance of the PAT was not discussed. Nautiyal et al. [8] performed normal and reverse running model tests of a centrifugal pump. They obtained the relationship of working conditions between the pump and the PAT. However, the normal and reverse running correlation mechanism from the perspective of microscopic internal flow was not investigated. Jain et al. [9] analysed the performance of a centrifugal pump under the PAT working condition via model test and optimized the impeller diameter and the rotational speed. Shinhama et al. [10] analysed radial thrust and runaway speed of a model of the pump and the PAT under the working conditions, and obtained the conversion coefficient between them. Bansal and Marshall [11] and Mirza [12] compared the connection modes and application environments of different PAT types and proposed applicable conditions of various schemes. Nautiyal et al. [13] analysed the PAT working condition of pump inversion by combining numerical simulation and model test, and proposed a performance prediction method of the PAT. Singh and Nestmann [14] sheared impeller blades under the PAT working condition, which increased the efficiency to some extent. On this basis, the optimized model was verified via experiment. Fernández et al. [15] confirmed that the pump performance could be estimated through PAT performance and verified multiple models. Yang et al. [16] investigated PAT under various rotational speeds via numerical simulation. They concluded that the flow rate and head conversion coefficient between the pump and the PAT decreased with the increase of rotational speed.

Changes on the structural parameters of an impeller, which is the core component of PAT, have a remarkable influence on its performance. Researchers have studied PAT performance under different parameters by combining numerical simulation and model test. For example, Singh and Nestmann [17] proposed a performance optimization method when a centrifugal pump was used as the PAT and verified the method by experimental test of three different rotational speeds. The accuracy could be controlled within $4 \%$. Himawanto and Tjahjana [18] explored the optimal working point of using pump as the PAT by changing the blade curvature. Chai et al. [19] designed a PAT model with four different blade outlet angles and concluded that the best performance was achieved when outlet angle of the blade was $45^{\circ}$. Miao et al. [20] proposed an optimization and design method under multiple working conditions of a PAT blade pattern based on a neural network with a genetic algorithm. The optimized PAT efficiency was 3\%-4\% higher than that of the original scheme.

Impellers with forward-curved blades and excellent hydraulic performance have been used extensively in the water turbine field. For example, Bai et al. [21] proposed a new design 
method for water turbine with forward-curved blades by combining the model test and computational fluid dynamics and displayed the hydraulic loss distribution in the water turbine and impeller. Wang et al. [22] designed an impeller with forward-curved blades and tested its external characteristics. The results showed that the efficiency of an impeller with forward-curved blades was remarkably higher than that of a traditional impeller with backward-curved blades and the efficiency curve was flatter. The variation laws of the outer diameter of the impeller with forward-curved blades and the blade outlet angle were also investigated. Yang et al. [23] performed a simulation and experimental study on forward- and backward-curved blades and concluded that the impeller with forward-curved blades was more suitable to the PAT.

In summary, researchers have conducted associated studies on the single-stage PAT model (no guide vane) or the fluid mechanics with an impeller having forward-curved blades. However, when the requirement of recovery power is high, like the centrifugal pump, the multi-stage PAT must adapt to the working conditions. In other words, the multi-stage PAT has additional guide vane compared with the single-stage PAT model. The above-mentioned results cannot reflect the actual performance of the PAT with guide vane. Thus, a PAT with a guide vane model is used as the research object in this study. The computational method of the PAT with guide vane model under multiple working conditions is constructed by combining numerical simulation and model test. Based on the structure of the impeller with forward-curved blades, the influences of the number of blades, blade outlet angle, and wrapping angle of the blade on the internal flow are discussed. Moreover, the optimal physical model of the blade under various parameters is constructed to optimize the hydraulic performance of the PAT. Research conclusions can provide reference for the optimization of cavitation performance of other blade-type hydraulic machinery.

The remainder of this study is organized as follows. Section 3 introduces the structural hydraulic model design and model test of a PAT. Section 4 investigates the optimization design of key geometric parameters and their influences on performance by analysing the internal flow characteristics of an impeller with forward-curved blades. Section 5 summarizes the conclusions.

\section{METHODOLOGY}

\subsection{Modelling and analysis}

A multilevel PAT model is used as the research object. The model is composed of an impeller with forward-curved blades, a guide vane, and a ring volute, as shown in Fig. 1. The design parameters are set as follows: flow rate $Q=200 \mathrm{~m}^{3} / \mathrm{h}$, head $H=100 \mathrm{~m}$, and speed $n=2950 \mathrm{r} / \mathrm{min}$. Table I lists the key geometric parameters.

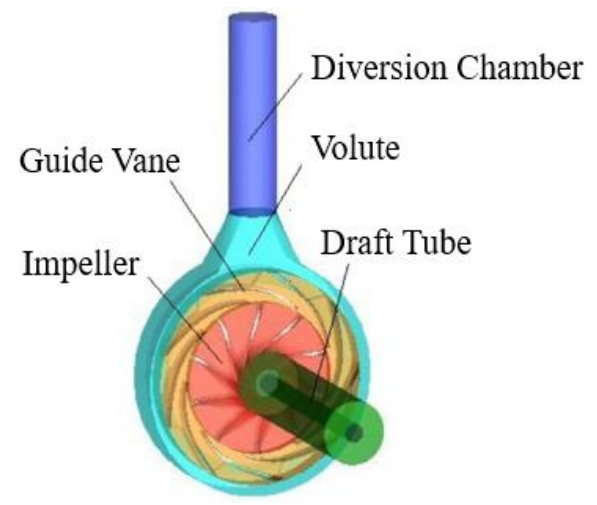

Figure 1: Water body map of the PAT with forward-curved blades. 
Table I: Main parameters of the impeller with forward-curved blades.

\begin{tabular}{|l|c|}
\hline \multicolumn{1}{|c|}{ Parameters } & Numerical values \\
\hline Inlet diameter of the impeller, $D_{2}(\mathrm{~mm})$ & 280 \\
\hline Outlet diameter of the impeller, $D_{1}(\mathrm{~mm})$ & 120 \\
\hline Wrapping angle of the blade, $\varphi\left(^{(}\right)$ & 35 \\
\hline Blade inlet angle, $\beta_{2}\left({ }^{\circ}\right)$ & 105 \\
\hline Blade outlet angle, $\alpha_{1}\left({ }^{\circ}\right)$ & 33.5 \\
\hline Number of blades, $Z$ & 11 \\
\hline
\end{tabular}

\subsection{Calculation method}

(1) Boundary conditions and meshing

The 3D incompressible fluid controlling equations are discretized using a finite volume method based on the ANSYS CFX 16.0 platform to expose the internal flow law of the PAT with guide vane. The controlling equations of the $3 \mathrm{D}$ turbulence numerical simulation involved the cavitation model of the two-phase flow mixed model, Reynolds-averaged Navier-Stokes equation, and shear stress transport $k-\omega$ turbulence model, which are highly suitable for fluid separation [24]. The discretion of the governing equations is used for volume control technique, and the equation diffusion term is the central difference scheme. The convective term uses a second-order upwind scheme. The equation solution is used a semiimplicit pressure coupling algorithm for separation. The pump inlet boundary is set as the total pressure inlet and outlet boundary is set as mass flow. Shear conditions on walls are set as no-slip condition, and the reference pressure is $0 \mathrm{~Pa}$. The energy transmission between the rotating (i.e., impeller) and static (i.e., guide vane) parts are adopted the "frozen rotor" connection. The calculation convergence standard is set to $10^{-5}$, and the medium uses $t=25^{\circ}$ water.

In this study, all the hydraulic parts are meshed using the ANSYS ICEM 16.0, and the $\mathrm{y}^{+}$ value is adjusted appropriately. The near-wall viscous sublayer that influences the key parts (i.e., blades and guide vane) of the hydraulic performance meets the requirement of $y^{+} \leq 5$, whereas the other flow parts meet the requirement of the near-wall viscous sublayer, that is, $\mathrm{y}^{+}<50$. Furthermore, the grid independence in the computational area of the water pump is verified [25]. Finally, the number of impeller grids, guide vane grids, and volute grids are 4.218 million, 3.254 million, and 5.815 million, respectively. The efficiency change is $1.02 \%$. Hence, the total number of grids in the pump model is suggested to be approximately 15.287 million.

(2) Model optimization method

Keeping the same parameters of PAT volute and guide vane, the influences of the three main parameters (i.e., $Z, \alpha_{1}$, and $\varphi$ ) of the impeller with forward-curved blades on the hydraulic performance and the corresponding optimization are discussed as follows.

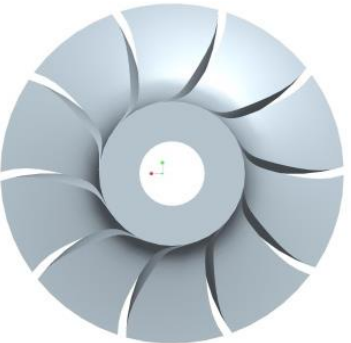

a) $Z=10$

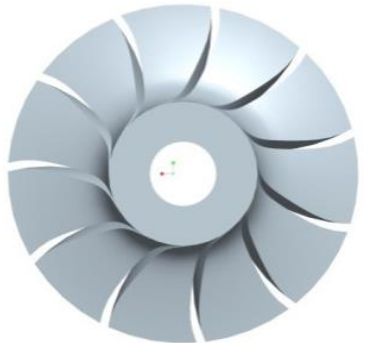

b) $Z=11$

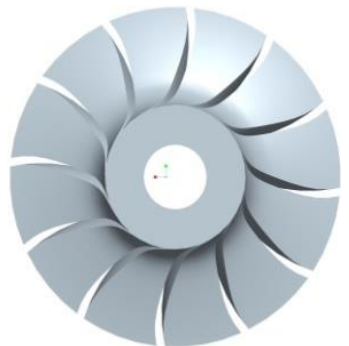

c) $Z=12$

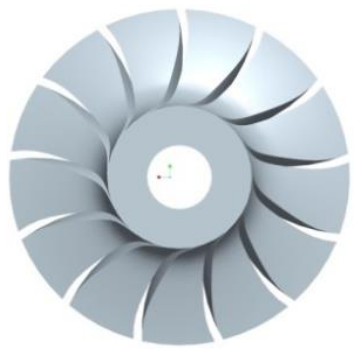

d) $Z=13$

Figure 2: 3D diagrams of the forward-curved blades with different numbers of blades and the fluid zone of the impeller. 
The number of blades can influence the PAT performance to some extent. Given the same parameters of the impeller with forward-curved blades, different numbers of blades (i.e., 10, 11,12 , and 13) are selected to perform the numerical simulation. The internal flow law is analysed. Fig. 2 shows the 3D diagrams of the blade.

The blade outlet angles at various flow rates of 325,450 , and $568 \mathrm{~m}^{3} / \mathrm{h}$ are calculated. The hydraulic characteristics of the PAT having the impeller with forward-curved blades under different blade outlet angles are discussed. The flow angle of fluid through the blade outlet is calculated as follows:

$$
\begin{aligned}
\tan \beta_{1}^{\prime} & =\frac{V_{m 1}}{u_{1}-V_{u 1}} \\
V_{m 1} & =\frac{Q}{\eta_{v} F_{1} \psi_{1}}
\end{aligned}
$$

where $\beta_{1}^{\prime}$ is the fluid flow angle of the outlet side, $v_{m 1}$ is the meridional velocity at the outlet, $u_{1}$ is the peripheral speed at the outlet side, $v_{u 1}$ is the peripheral component of velocity at the outlet side, $\eta_{v}$ is the volume efficiency, $F_{1}$ is the discharge section area of the outlet side, and $\Psi_{1}$ is the excretion coefficient of the blade at the outlet side. The draft tube is the cone-shaped suction chamber of the pump; thus, the ideal outlet liquid has no circular rector. Thus, $v_{u 1}=0$.

After the calculation, the outlet blade angles of the flow lines in the middle of the blade in various schemes are $33.5^{\circ}, 47^{\circ}$, and $53.7^{\circ}$. The influences of the three angles of the blade outlet on the hydraulic performance are discussed. The impeller with forward-curved blades is designed using the wrapping angles of $35^{\circ}, 45^{\circ}$, and $55^{\circ}$ due to the different distortion directions of the blades. Fig. 3 shows the 3D diagrams of the blade and water.

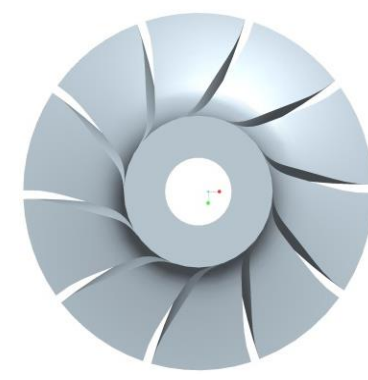

a) $\varphi=35^{\circ}$

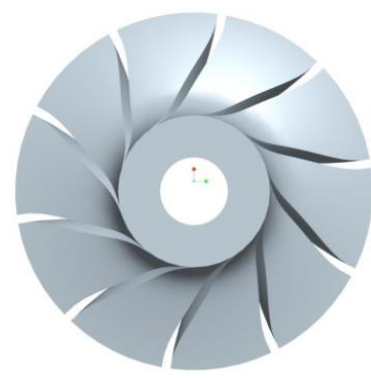

b) $\varphi=45^{\circ}$

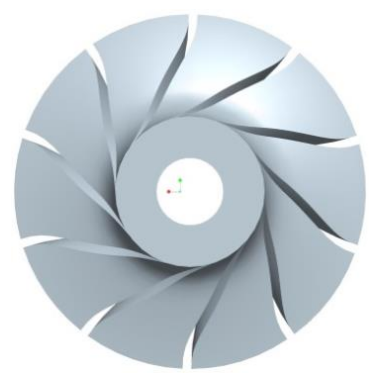

c) $\varphi=55^{\circ}$.

Figure 3: 3D diagrams of the forward-curved blades with different blade angles and the fluid zone of the impeller.

\subsection{Construction of the model test}

The PAT test bench is constructed according to China's national standards (GB3216-2005). It consists of a model PAT, an overrunning clutch, an electric motor, a centrifugal pump, throttle valves, bypass valves, and a pool. The test bench diagram is shown in Fig. 4. It consists of the pump and motor and PAT. Energies recovered by the PAT are used to drive the pressure supply pump. The PAT and motor are connected by the overrunning coupler, which prevent the asynchronous phenomenon between the PAT and motor. During the test, firstly, valve 3 is closed and valve 2 is completely opened. Secondly, valve 1 is adjusted to ensure the stable operation of the pressure supply pump in the high-efficiency area. Thirdly, valve 1 is adjusted continuously to obtain different heads. Finally, the openings of valves 2 and 3 are coordinated to adjust the flow rate of the PAT to obtain external characteristic curves of the PAT at a constant rotational speed. 


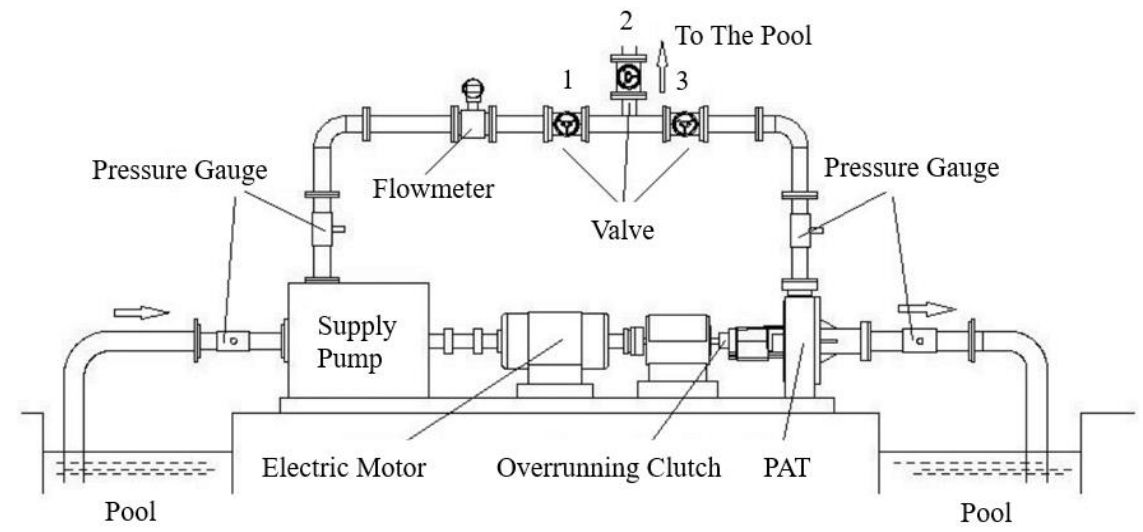

Figure 4: Test bench diagram.

\section{RESULT ANALYSIS AND DISCUSSION}

\subsection{Performance comparison}

The performance parameters are acquired from the test. The tested relationship curves among the head, efficiency, power, and flow rate of the PAT are drawn and compared with the simulation result, as shown in Fig. 5. The simulation value agrees with the test value. The numerical calculation approximately is simplified the fluid domain to a certain extent, and only hydraulic loss is considered. Thus, the simulation results are higher than the experimental results. However, the error is within the engineering allowable range.

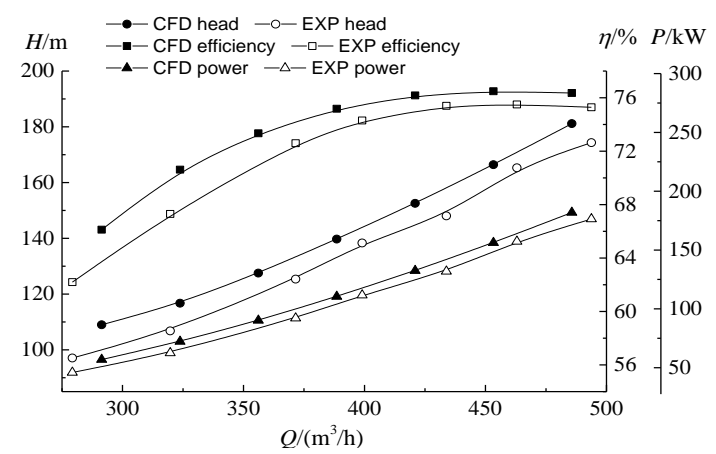

Figure 5: Comparison of the simulation and experimental results.

\subsection{Influence of the number of blades}

Figs. 6 and 7 show the numerical results of the PAT with four different blade numbers (i.e. $10,11,12,13)$ while the other parameters keep the same.

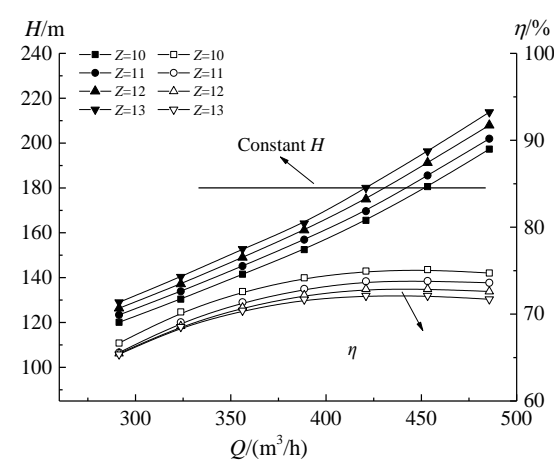

a) $Q-H$ and $Q-\eta$

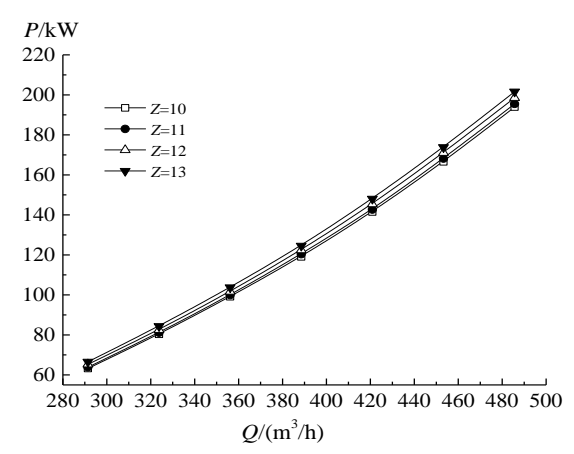

b) $Q-P$

Figure 6: External characteristic curves of different number of blades of the PAT at a constant speed. 
Table II: Optimum operating point parameters of the PAT with forward-curved blades for different numbers of blade.

\begin{tabular}{|c|c|c|c|c|}
\hline$Z$ & $Q\left(\mathrm{~m}^{3} / \mathrm{h}\right)$ & $H(\mathrm{~m})$ & $\eta(\%)$ & $P(\mathrm{~kW})$ \\
\hline 10 & 450 & 180.6 & 75.12 & 166.19 \\
\hline 11 & 450 & 185.6 & 73.81 & 167.81 \\
\hline 12 & 450 & 191.2 & 72.89 & 170.72 \\
\hline 13 & 450 & 196.3 & 72.11 & 173.40 \\
\hline
\end{tabular}

As shown in Fig. 6 a, the head of the PAT with different numbers of blades have the same variation tendency with the increase of flow rate. Given the same flow rate, the head of the PAT is large when additional blades are used. At the optimal flow point, the head difference between the two adjacent numbers of the blades is approximately $5 \mathrm{~m}$, which remains constant. The number of blades can slightly influence the efficiency of the PAT. The difference value of the efficiency between the two adjacent numbers of the blades is approximately $1 \%$, and the aerodynamic efficiency between blades is approximately $3 \%$. When other conditions are constant in Table II, the changes in the number of blades in a certain range can slightly affect the optimal flow rate of the PAT. As shown in Fig. $6 \mathrm{~b}$, variations of the recovery power with different numbers of blades are similar to the variation tendencies of the head. The difference values between the recovery powers are similar to the efficiency changes. Given the fixed pressure at the PAT inlet, for fewer blades, it needs larger flow rate to achieve the maximum recovery head, meanwhile, the efficiency is higher, the recovery power is larger. The PAT model with 10 blades achieves the highest efficiency and recovery power under the same head and obtains the best hydraulic performance.

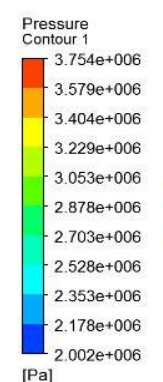

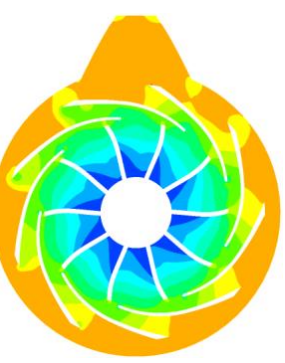

a) $Z=10$

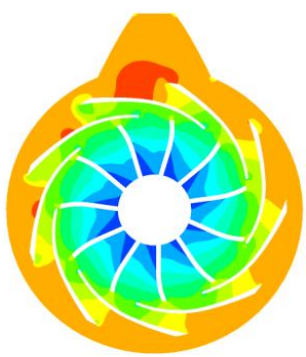

b) $Z=11$

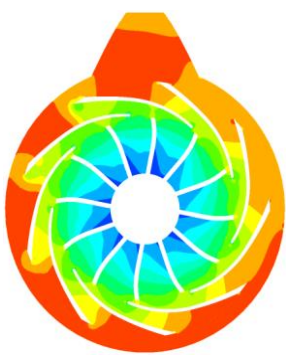

c) $Z=12$

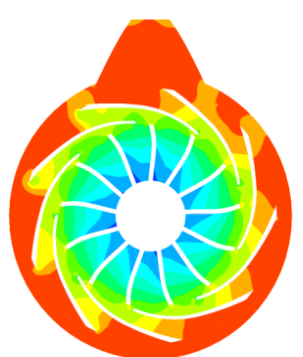

d) $Z=13$

Figure 7: Pressure contours of the middle section of the PAT impeller with different numbers of blades.

The static pressure distribution in the middle cross-section of the turbine model is shown in Fig. 7. The high-pressure area of the PAT is concentrated in the volute. The numerical distribution of the PAT pressure with four different numbers of blades conforms to the external characteristic head curves. The pressure distribution in the guide vane and impeller is relatively uniform. Low-pressure areas in the impeller concentrated on the suction side of the blade. When the number of blades is smaller, the low-pressure area in the impeller is larger.

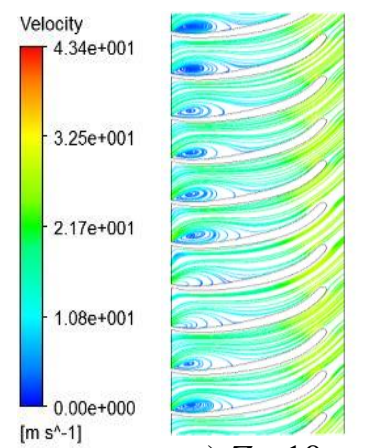

a) $Z=10$

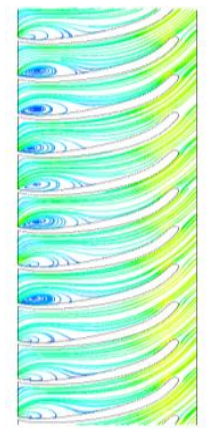

b) $Z=11$

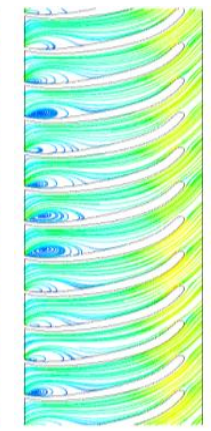

c) $Z=12$

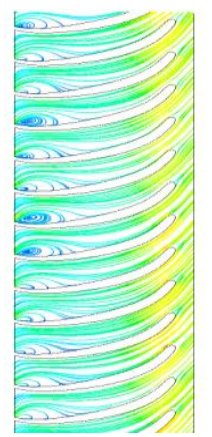

d) $Z=13$

Figure 8: Blade streamlines diagrams of $z=0.5$ of the PAT impeller with different numbers of blades. 
A large eddy is formed at the impeller inlet (Fig. 8) which is possibly caused by the large distance between blades, short flow channels, and angle of attack at the impeller inlet. The PAT with four different numbers of blades all exist an inlet eddy, and the flow in other areas of the impeller is relatively uniform. With increasing number of blades, the strength of the inlet eddy reduces to some extent.

By the analysis of PAT models with four numbers of blades, the highest efficiency of the PAT is achieved when the number of blades is 10 , which can also result in a stable internal flow.

\subsection{Influence of the blade outlet angle}

The blade outlet angle is constantly changing from the hub to the shroud. The blade outlet angle $\left(\alpha_{1}\right)$ at the middle streamline of the blade represents the blade outlet angle. Without changing other parameters, the numerical simulations are performed using different blade outlet angles (i.e., $33.5^{\circ}, 47^{\circ}, 53.7^{\circ}$ ) while keeping 10 blades.

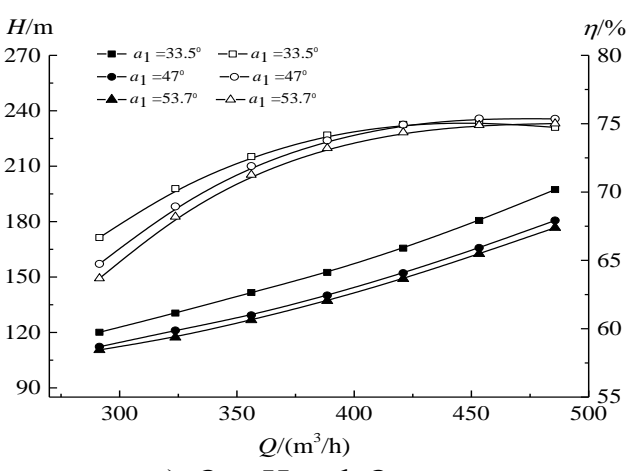

a) $Q-H$ and $Q-\eta$

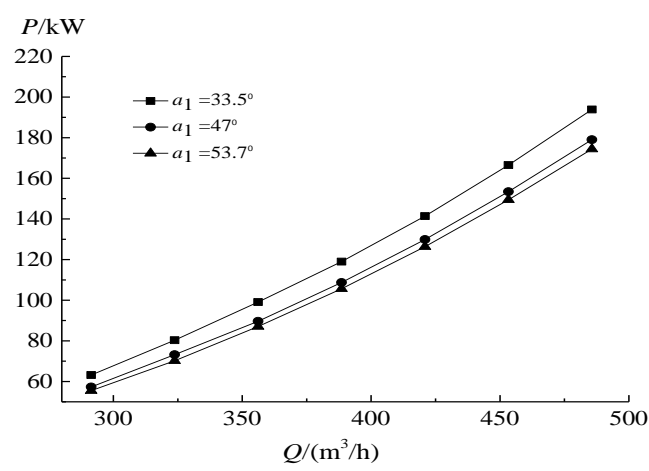

b) $Q-P$

Figure 9: External characteristic curves at a constant speed of the PAT with different exit angles of the blade.

Table III: Optimum operating point parameters of the PAT with different blade outlet angles.

\begin{tabular}{|c|c|c|c|c|}
\hline$\alpha_{1}$ & $Q\left(\mathrm{~m}^{3} / \mathrm{h}\right)$ & $H(\mathrm{~m})$ & $\eta(\%)$ & $P(\mathrm{~kW})$ \\
\hline$\alpha_{1}=33.5^{\circ}$ & 450 & 180.6 & 75.12 & 166.5 \\
\hline$\alpha_{1}=47^{\circ}$ & 450 & 165.8 & 75.38 & 153.4 \\
\hline$\alpha_{1}=53.7^{\circ}$ & 486 & 176.7 & 75.01 & 174.4 \\
\hline
\end{tabular}

As shown in Fig. 9 a, as the flow rate increases, the head of the PAT gradually increases, whereas the efficiency initially increases and then decreases. With different blade outlet angles, the PAT heads have equal growth tendency with the increase of the flow rate. The PAT with large outlet angle requires a small head. The calculated flow rate with the blade outlet angle of $33.5^{\circ}$ is $325 \mathrm{~m}^{3} / \mathrm{h}$, and the efficiency is the highest at a small flow rate. The calculated flow rate with the blade outlet angle of $47^{\circ}$ is $450 \mathrm{~m}^{3} / \mathrm{h}$, and the efficiency is the highest at $450 \mathrm{~m}^{3} / \mathrm{h}$. The calculated flow rate with the outlet angle of $53.7^{\circ}$ is $568 \mathrm{~m}^{3} / \mathrm{h}$, which exceeded the optimal flow rate in PAT operation, and the total efficiency is smaller than that with the outlet angle of $47^{\circ}$. As shown in Fig. $9 \mathrm{~b}$, the variation trends of the recovery power with blade outlet angle are similar to the variation laws of the head. At the same flow rate, the PAT with small blade outlet angle achieves high recovery power. As the blade outlet angle increases, the flow rate at the optimal efficiency point of the model turbine is $450 \mathrm{~m}^{3} / \mathrm{h}$ (Table III). When the outlet angle continues to increase, the flow rate at the optimal efficiency point will transfer to the large flow rate. The flow rate at the optimal efficiency point is $486 \mathrm{~m}^{3} / \mathrm{h}$ when the outlet angle is $53.7^{\circ}$. In other fixed conditions, the changes of the blade outlet angle in a certain range easily cause shifts of the turbine to the optimal working condition point. 


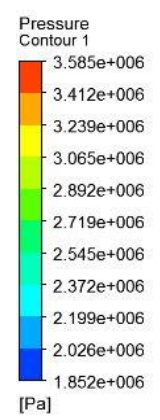

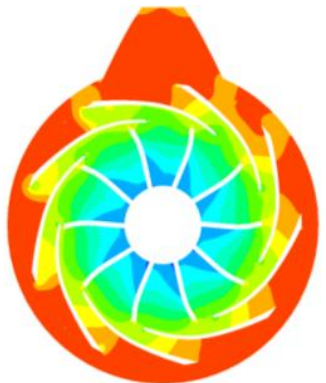

a) $\alpha_{1}=33.5^{\circ}$

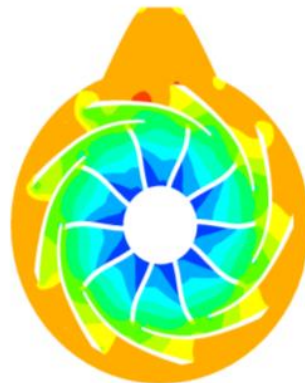

b) $\alpha_{1}=47^{\circ}$

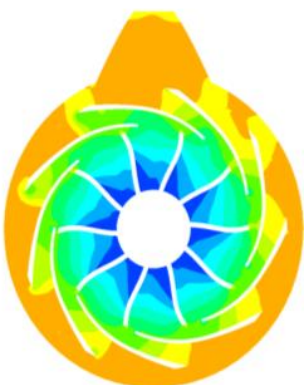

c) $\alpha_{1}=53.7^{\circ}$

Figure 10: Pressure cloud diagrams of the middle section of the PAT impeller with different blade outlet angles.

The pressure distribution of the PAT model with three blade outlet angles (Fig. 10) conforms to the external characteristic of the head curves. The pressure distribution in the volute is relatively uniform. Two pressure distributions of the PAT at the left and right sides of the guide vane under three different blade outlet angles are observed. The low-pressure region at the left side of the guide vane is larger than that at the right side due to the resistance at the left side. This region is generally concentrated on the suction side of the blade. As the blade outlet angle increases, the low-pressure area gradually increases.
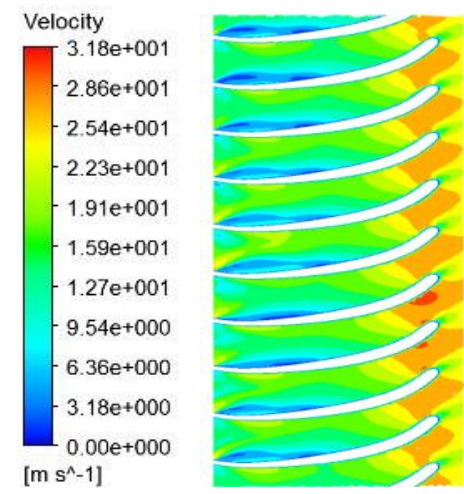

a) $\alpha_{1}=33.5^{\circ}$

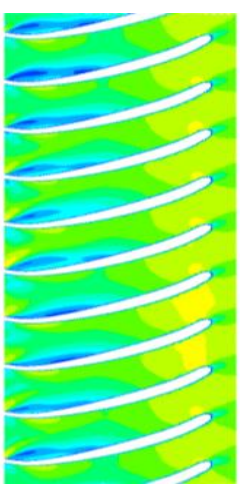

b) $\alpha_{1}=47^{\circ}$

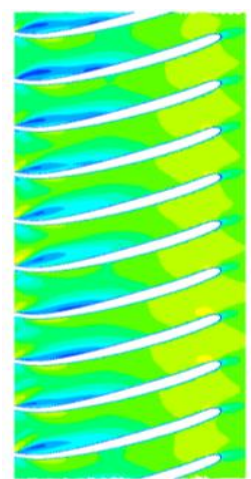

c) $\alpha_{1}=53.7^{\circ}$

Figure 11: Blade velocity cloud maps of $z=0.5$ of the PAT impeller with different blade outlet angles.

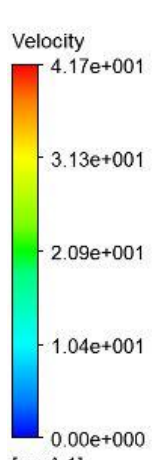

$\left[\mathrm{m} \mathrm{s} \mathrm{s}^{\wedge}-1\right]$

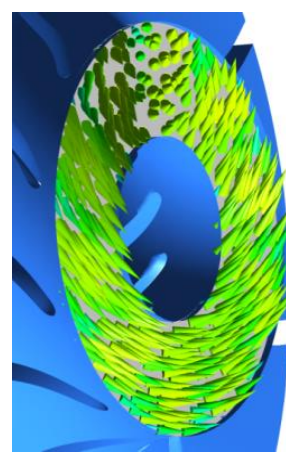

a) $\alpha_{1}=33.5^{\circ}$

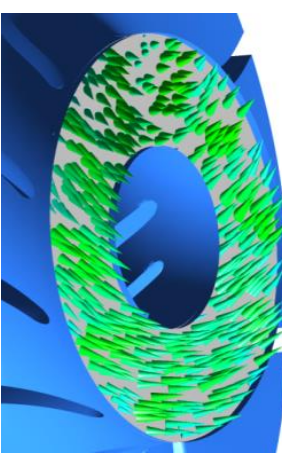

b) $\alpha_{1}=47^{\circ}$

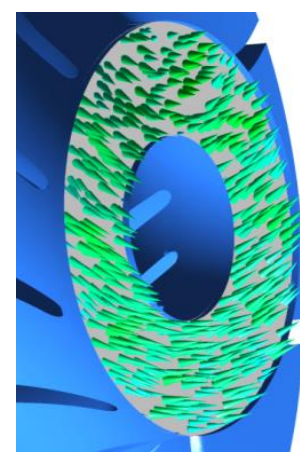

c) $\alpha_{1}=53.7^{\circ}$

Figure 12: Velocity vector diagrams of the exit section of $L=40 \mathrm{~mm}$ from the cross section of the impeller at the outlet angle of different blades.

As shown in Fig. 11, the low-pressure region of the eddy at the blade inlet decreases with the increase of the blade outlet angle; this indicates the effect of the outlet angle on the internal eddy intensity. The throat area at the impeller outlet changes with the changes of the outlet angle. When the outlet angle is $33.5^{\circ}$, the internal throat area is the smallest. In the high-pressure area at the blade tail, the throat area expands gradually with the increase of the 
outlet angle and the velocity at the blade tail decrease. The velocity at the blade tail is the smallest when the outlet angle is $53.7^{\circ}$.

The 3D PAT velocity with small outlet angle is higher than those with other blade outlet angles (Fig. 12), and the velocity direction varies. The included angle between the outlet velocity and axis of the PAT with small outlet angle is larger, which indicates the higher outlet velocity spinor of the impeller. The comprehensive analysis shows that under the same head the PAT model with $47^{\circ}$ blade outlet angle achieves the highest efficiency, power and the best hydraulic performance.

\subsection{Influence of the wrapping angle of the blade}

As shown in Fig. 13 a, the head needed by the PAT under three wrapping angles of the blade are similar and have an intersection point at a small flow rate. The efficiency curves also have an intersection point at a small flow rate, and the growth tendencies of the head with increasing flow are same. When the flow rate is smaller than $325 \mathrm{~m}^{3} / \mathrm{h}$, the PAT head under $35^{\circ}$ wrapping angle of the blade is slightly higher than those with the other two wrapping angles of the blade. When the flow rate is higher than $325 \mathrm{~m}^{3} / \mathrm{h}$, the PAT head under $55^{\circ}$ wrapping angle of the blade gradually exceeds other two wrapping angles of the blade. Generally, the head needed by the PAT is positively related to the large wrapping angle of the blade. When the flow rate is smaller than $356 \mathrm{~m}^{3} / \mathrm{h}$, the PAT efficiency under $55^{\circ}$ is higher than that under $35^{\circ}$. When the flow rate is smaller than $308 \mathrm{~m}^{3} / \mathrm{h}$, the PAT efficiency under $55^{\circ}$ is higher than that under $45^{\circ}$. When the flow rate is higher than $356 \mathrm{~m}^{3} / \mathrm{h}$, the PAT efficiency under $45^{\circ}$ is the highest.

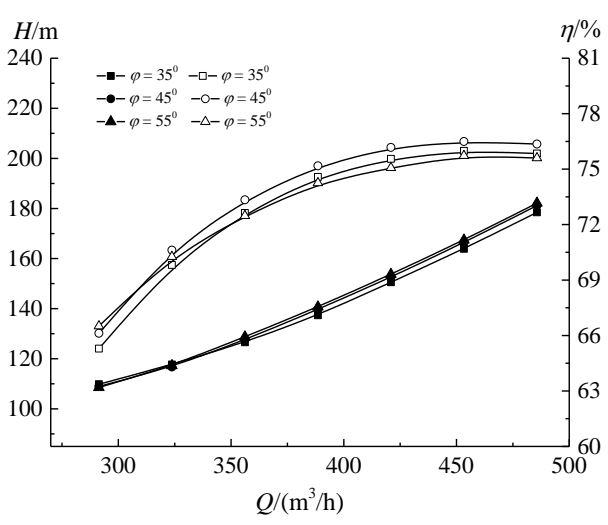

a) $Q-H$ and $Q-\eta$

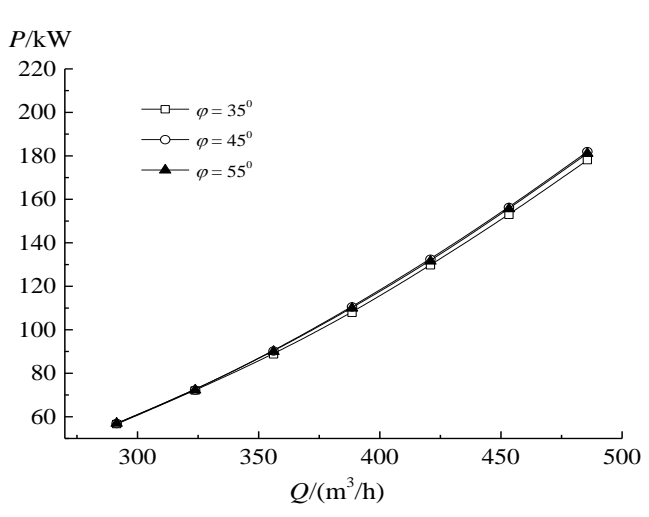

b) $Q-P$

Figure 13: External characteristic curves at a constant speed of the PAT with different wrapping angles of the blade.

Table IV: Optimum operating point parameters of the PAT with forward-curved blades under different wrapping angles of the blade.

\begin{tabular}{|c|c|c|c|c|}
\hline$\varphi$ & $Q\left(\mathrm{~m}^{3} / \mathrm{h}\right)$ & $H(\mathrm{~m})$ & $\eta(\%)$ & $P(\mathrm{~kW})$ \\
\hline $35^{\circ}$ & 450 & 163.9 & 75.98 & 152.8 \\
\hline $45^{\circ}$ & 450 & 166.4 & 76.49 & 156.3 \\
\hline $55^{\circ}$ & 450 & 167.4 & 75.72 & 155.6 \\
\hline
\end{tabular}

As shown in Table IV, when the wrapping angle of the blade increases from $35^{\circ}$ to $55^{\circ}$, the flow rate at the optimal efficiency points of the PAT is the same $\left(450 \mathrm{~m}^{3} / \mathrm{h}\right)$. As shown in Fig. $13 \mathrm{~b}$, the variation tendencies of power with different wrapping angles of the blade are similar to that of the head. At the same flow rate, the difference value is small. The PAT with $45^{\circ}$ wrapping angle of the blade achieves the highest efficiency and recovery power, as well as the best hydraulic performance under the same head. 


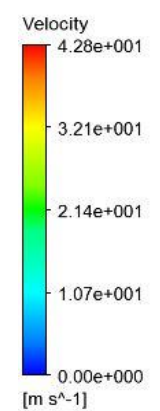

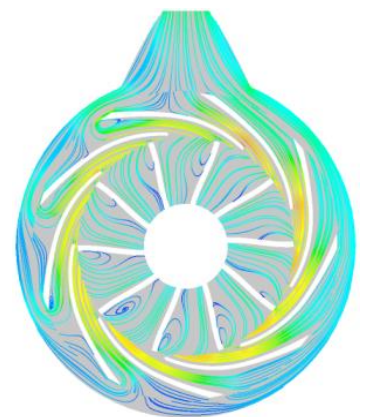

a) $\varphi=35^{\circ}$

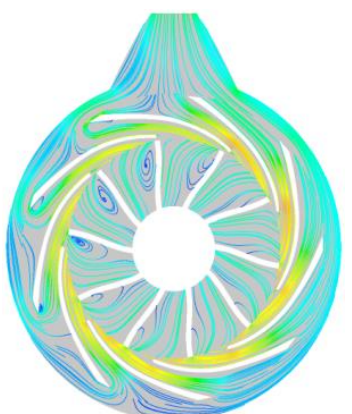

b) $\varphi=45^{\circ}$

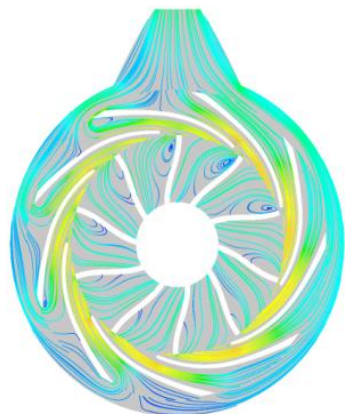

c) $\varphi=55^{\circ}$

Figure 14: Streamline diagrams in the middle section of the PAT model impeller under different wrapping angles of the blade.

As shown in Fig. 14, the blade twist gradually increases with the wrapping angle of the blade increasing. In the volute and guide vane, the streamline distribution slightly varies. In the impeller, the eddy at the blade inlet is highly susceptible to the wrapping angle of the blade. The eddy distribution in circumferential direction along the impeller is not uniform. When the wrapping angle of the blade is $35^{\circ}$, several large eddies ware far from the volute inlet. At $45^{\circ}$ and $55^{\circ}$, large eddies are close to the volute outlet. Generally, the eddy in the impeller is small, and the distribution is relatively uniform at $45^{\circ}$.
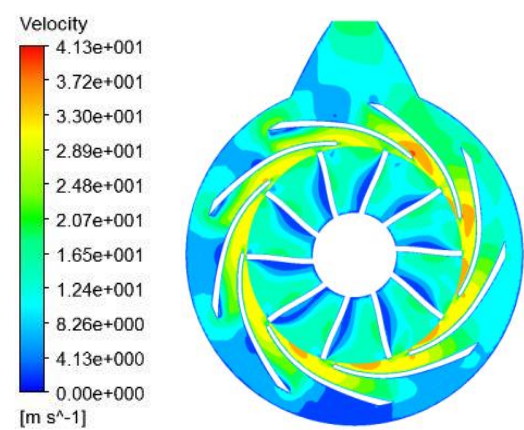

a) $\varphi=35^{\circ}$

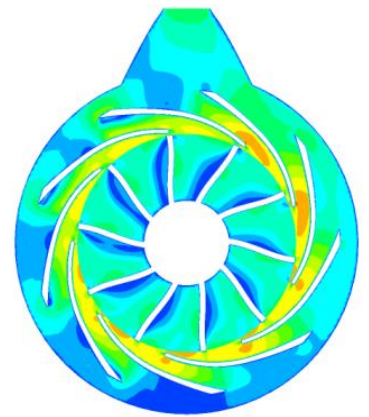

b) $\varphi=45^{\circ}$

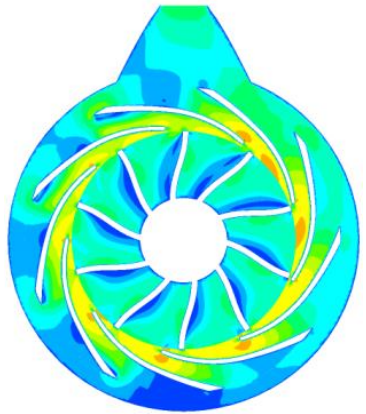

c) $\varphi=55^{\circ}$

Figure 15: Velocity images in the middle section of the PAT model impeller under different wrapping angles of the blade.

As shown in Fig. 15, the high-velocity region at the right side of the guide vane decreases to some extent with the blade twisting severely. This result indicates that the flow at the right side of the volute slightly decreases, and the flow rates at the left and right sides of the ring volute are similar, thereby influencing the velocity distribution in the impeller. The lowvelocity region in the blade pressure side is close to the blade wall when the wrapping angle of the blade increases from $35^{\circ}$ to $55^{\circ}$. At $45^{\circ}$, the low-pressure region in the impeller is uniform and stable. Hence, the PAT model with 10 blades, $47^{\circ}$ blade outlet angle, and $45^{\circ}$ wrapping angle of the blade achieves the best comprehensive performance.

\section{CONCLUSION}

Theoretical analysis, numerical simulation, and model test were combined to disclose the variation law of the internal flow characteristics of the PAT with guide vane and optimize the running efficiency and recovery power. The impeller with forward-curved blades and some critical geometric parameters (e.g., number of blades, blade outlet angle, and wrapping angle of the blade) that influence its hydraulic performance were analysed. The following conclusions could be drawn. 
(1) By comparing the results of numerical simulation with the model test considering the designed working condition, the PAT head, efficiency, and recovery power error can all meet requirements of engineering applications. The numerical results can accurately predict the actual flow state of the PAT model.

(2) The number of blades can remarkably influence the PAT efficiency. With the increase of the number of blades, the head required for the PAT increases gradually, whereas the efficiency decreases. As the blade outlet angle increases, the optimal working condition point of the PAT moves toward the large flow rate. With respect to the efficiency at the optimal working point, an optimal angle exists. The PAT with a large wrapping angle of the blade requires a large head. The turbine efficiency with $45^{\circ}$ wrapping angle of the blade is $76.49 \%$ and achieves the highest recovery power with the same head.

(3) The numerical simulation on the PAT model of the impeller with forward-curved blades is performed. The key geometric parameters are optimized and designed to obtain an excellent hydraulic model. The optimized parameters are 10 blades, $47^{\circ}$ blade outlet angle, and $45^{\circ}$ wrapping angle of the blade.

This study has combined numerical simulation and experiments, established the internal relationships among the number of blades, blade outlet angle, and wrapping angle of the blade of the impeller with forward-curved blades. The results are important for guiding the design and performance optimization of multi-stage PAT models. However, considering the lack of visible test data on the internal flow field of the PAT model with a guide vane, a contrastive analysis on the geometric parameters should be implemented in future works by combining the particle image velocity measurement technology, which can systematically optimize the hydraulic performance of the impeller with forward-curved blades.

\section{REFERENCES}

[1] Bi, Z.-G.; Yang, J.-H.; Zhao, W.-Y. (2014). Status of research of hydraulic turbines for energy recovery, Fluid machinery, Vol. 31, No. 8, 41-45,doi:10.3969/j.issn.1005-0329.2014.08.009

[2] Chen, K.; Yang, S.-S.; Wang, T.; Kong, F.; Yuan, X. (2016). Numerical research on impeller diameter influencing the performance of hydraulic turbine with forward-curved blades, Journal of Mechanical Engineering, Vol. 52, No. 22, 198-204, doi:10.3901/JME.2016.22.198

[3] Carravetta, A.; Antipodi, L.; Golia, U.; Fecarotta, O. (2017). Energy saving in a water supply network by coupling a pump and a pump as turbine (PAT) in a turbopump, Water, Vol. 9, No. 1, Paper 62, 14 pages, doi: $10.3390 /$ w9010062

[4] De Marchis, M.; Milici, B.; Volpe, R.; Messineo, A. (2016). Energy saving in water distribution network through pump as turbine generators: economic and environmental analysis, Energies, Vol. 9, No. 11, Paper 877, 15 pages, doi:10.3390/en9110877

[5] Lydon, T.; Coughlan, P.; McNabola, A. (2017). Pump-as-turbine: characterization as an energy recovery device for the water distribution network, Journal of Hydraulic Engineering, Vol. 143, No. 8, Paper 04017020, doi:10.1061/(ASCE)HY.1943-7900.0001316

[6] Derakhshan, S.; Nourbakhsh, A. (2008). Theoretical, numerical and experimental investigation of centrifugal pumps in reverse operation, Experimental Thermal and Fluid Science, Vol. 32, No. 8, 1620-1627, doi:10.1016/j.expthermflusci.2008.05.004

[7] Derakhshan, S.; Mohammadi, B.; Nourbakhsh, A. (2009). Efficiency improvement of centrifugal reverse pumps, Journal of Fluids Engineering, Vol. 131, No. 2, Paper 021103, 9 pages, doi: $10.1115 / 1.3059700$

[8] Nautiyal, H.; Varun, V.; Kumar, A.; Sanjay, Y. (2011). Experimental investigation of centrifugal pump working as turbine for small hydropower systems, Energy Science and Technology, Vol. 1, No. 1, 79-86, doi:10.3968/j.est.1923847920110101.006

[9] Jain, S. V.; Swarnkar, A.; Motwani, K. H.; Patel, R. N. (2015). Effects of impeller diameter and rotational speed on performance of pump running in turbine mode, Energy Conversion and Management, Vol. 89, 808-824, doi:10.1016/j.enconman.2014.10.036 
[10] Shinhama, H.; Fukutomi, J.; Nakase, Y.; Chin, Y.; Kuwauchi, T.; Miyauchi, S. (1999). Study on reverse running pump turbine, Transactions of the Japan Society of Mechanical Engineers Series $B$, Vol. 65, No. 638, 3399-3405, doi:10.1299/kikaib.65.3399

[11] Bansal, P.; Marshall, N. (2010). Feasibility of hydraulic power recovery from waste energy in bio-gas scrubbing processes, Applied Energy, Vol. 87, No. 3, 1048-1053, doi:10.1016/ j.apenergy.2009.09.010

[12] Mirza, S. (2008). Reduction of energy consumption in process plants using nanofiltration and reverse osmosis, Desalination, Vol. 224, No. 1-3, 132-142, doi:10.1016/j.desal.2007.04.084

[13] Nautiyal, H.; Varun, G.; Kumar, A. (2010). Reverse running pumps analytical, experimental and computational study: a review, Renewable and Sustainable Energy Reviews, Vol. 14, No. 7, 2059-2067, doi:10.1016/j.rser.2010.04.006

[14] Singh, P.; Nestmann, F. (2011). Internal hydraulic analysis of impeller rounding in centrifugal pumps as turbines, Experimental Thermal and Fluid Science, Vol. 35, No. 1, 121-134, doi:10.1016/j.expthermflusci.2010.08.013

[15] Fernández, J.; Blanco, E.; Parrondo, J.; Stickland, M. T.; Scanlon, T. J. (2004). Performance of a centrifugal pump running in inverse mode, Proceedings of the Institution of Mechanical Engineers, Part A: Journal of Power \& Energy, Vol. 218, No. 4, 265-271, doi: $10.1243 / 0957650041200632$

[16] Yang, S.-S.; Li, Q.; Huang, Z.-P.; Kong, F.-Y.; Shi, H.-X. (2013). Research on different specific speed pumps used as turbines, Transactions of the Chinese Society for Agricultural Machinery, Vol. 44, No. 3, 69-72, doi:10.6041/j.issn.1000-1298.2013.03.014

[17] Singh, P.; Nestmann, F. (2010). An optimization routine on a prediction and selection model for the turbine operation of centrifugal pumps, Experimental Thermal and Fluid Science, Vol. 34, No.2, 152-164, doi:10.1016/j.expthermflusci.2009.10.004

[18] Himawanto, D. A.; Tjahjana, D. D. D. P.; Hantarum (2017). Experimental study on optimization of curvature blade impeller pump as turbine which functioned as power plant picohydro, AIP Conference Proceedings, Vol. 1788, Paper 030008, 7 pages, doi:10.1063/1.4968261

[19] Chai, L.-P.; Shen, L.-B.; Liang, H.-B.; He, Y.-J.; Huang, M.-K. (2012). The performance simulation and experiment of high pressure hydraulic turbine, China Rural Water and Hydropower, Vol. 2012, No. 5, 125-128

[20] Miao, S.-C.; Yang, J.-H.; Wang, X.-H.; Li, J.-C.; Li, T.-L. (2015). Blade pattern optimization of the hydraulic turbine based on neural network and genetic algorithm, Journal of Aerospace Power, Vol. 30, No. 8, 1918-1925, doi:10.13224/j.cnki.jasp.2015.08.015

[21] Bai, Y.; Kong, F.; Yang, S.; Chen, K.; Dai, T. (2017). Effect of blade wrap angle in hydraulic turbine with forward-curved blades, International Journal of Hydrogen Energy, Vol. 42, No. 29, 18709-18717, doi:10.1016/j.ijhydene.2017.04.185

[22] Wang, T.; Kong, F.-Y.; Yang, S.-S.; Chen, K.; Xu, R.-J. (2015). Effects of blade angle distributions on performance of pump as turbine, Transactions of the Chinese Society for Agricultural Machinery, Vol. 46, No. 10, 75-80, doi:10.6041/j.issn.1000-1298.2015.10.012

[23] Yang, S.-S.; Zhang, H.; Kong, F.-Y.; Xu, R.-J. (2016). Analysis on blades curved shape of centrifugal pump as turbine, Journal of Shanghai Jiaotong University, Vol. 50, No. 9, 1467-1472 +1479 , doi: $10.16183 /$ j.cnki.jsjtu.2016.09.019

[24] Li, X.; Jiang, Z.; Zhu, Z.; Si, Q.; Li, Y. (2018). Entropy generation analysis for the cavitating head-drop characteristic of a centrifugal pump, Proceedings of the Institution of Mechanical Engineers, Part C: Journal of Mechanical Engineering Science, in Press, 10 pages, doi: $10.1177 / 0954406217753458$

[25] Yang, J.; Pavesi, G.; Liu, X.; Xie, T.; Liu, J. (2018). Unsteady flow characteristics regarding hump instability in the first stage of a multistage pump-turbine in pump mode, Renewable Energy, Vol.127, 377-385, doi:10.1016/j.renene.2018.04.069 